\title{
Transcatheter retrieval of embolized catheter using venovenous loop in a neonate
}

\author{
Birgül Varan ${ }^{1}$, Kahraman Yakut ${ }^{1}$, Ali Harman ${ }^{2}$ \\ Departments of ${ }^{1}$ Pediatric Cardiology and ${ }^{2}$ Interventional Radiology, Başkent University Faculty of Medicine, Ankara, \\ Turkey. E-mail: kahramanyakut@gmail.com \\ Received: 25th March 2017, Revised: 6th May 2017, Accepted: 1st June 2017
}

\begin{abstract}
SUMMARY: Varan B, Yakut K, Harman A. Transcatheter retrieval of embolized catheter using venovenous loop in a neonate. Turk J Pediatr 2018; 60: 113-115.

Umbilical venous catheters are reliable in providing adequate medical care for premature babies however complications include infections, bleeding, thrombosis, catheter occlusion and migration of a piece of catheter to other parts in the body. Here, we present a case with migration of a piece of umbilical venous catheter which has been trapped between hepatic vein and right upper pulmonary vein causing apnea episodes, recurrent respiratory symptoms, need for mechanical ventilation and increase of acute phase reactants. The embolized catheter was retrieved successfully via percutaneous route.
\end{abstract}

Key words: umbilical catheter, newborn, complication, treatment.

Umbilical venous catheters are commonly used in newborn intensive care units in recent years, particularly in premature babies with very low birth weight. It is known that umbilical venous catheters may lead to many complications during the acute and chronic period. In order to minimize these complications, care should be taken during insertion and removal of the catheter, it should be checked if it is in place or not after insertion, then should be followedup with certain intervals. Our purpose in presenting this case is to discuss frequently observed complications with umbilical venous catheters such as infection, bleeding, thrombosis as well as the rupture of catheter piece which is a rare complication due to technical methods and materials used during catheter fixation, its migration to any part of the body and its treatment in light of the current literature.

\section{Case Report}

Thirty week-old baby girl who was born $1500 \mathrm{~g}$ in weight from the fifth pregnancy of a 36-year-old mother was intubated due to respiratory distress and cyanosis and followedup on mechanical ventilation. Her birth weight and birth length was at 25-50th percentile, she had tachypnea and intercostal retractions. Due to respiratory distress syndrome compatible findings found on the chest radiography of the patient, surfactant treatment was applied once. She was extubated on the 5th day after birth and put on nasal continuous positive airway pressure. She received gentamycin and clindamycin for 10 days and ampicillin for 14 days. On the 16th day after birth, upon deterioration of her condition and beginning of respiratory distress findings, the baby was re-intubated and connected to a mechanical ventilator. The C-reactive protein level was elevated. Lumbar puncture was performed and cerebrospinal fluid examination was normal; vancomycin and meropenem treatment as well as intravenous immunoglobulin treatment were initiated for sepsis for 3 days ( $5 \mathrm{mg} / \mathrm{kg} /$ dose). Vancomycin and meropenem treatments were completed and stopped on the 18th day. In evaluations of the patient whose respiratory distress continued on the 34th day after birth while on nasal continuous positive airway pressure, existence of a foreign body extending from umbilical vein to right lung upper lobe on chest X-ray was identified. It was verified by CT scan of patient's entire abdomen and lung. She was taken into surgery by pediatric surgeons and the piece belonging to navel catheter was attempted to be removed, however this was unsuccessful. The patient was referred to another center where transcatheter removal of the catheter was attempted. This was also 


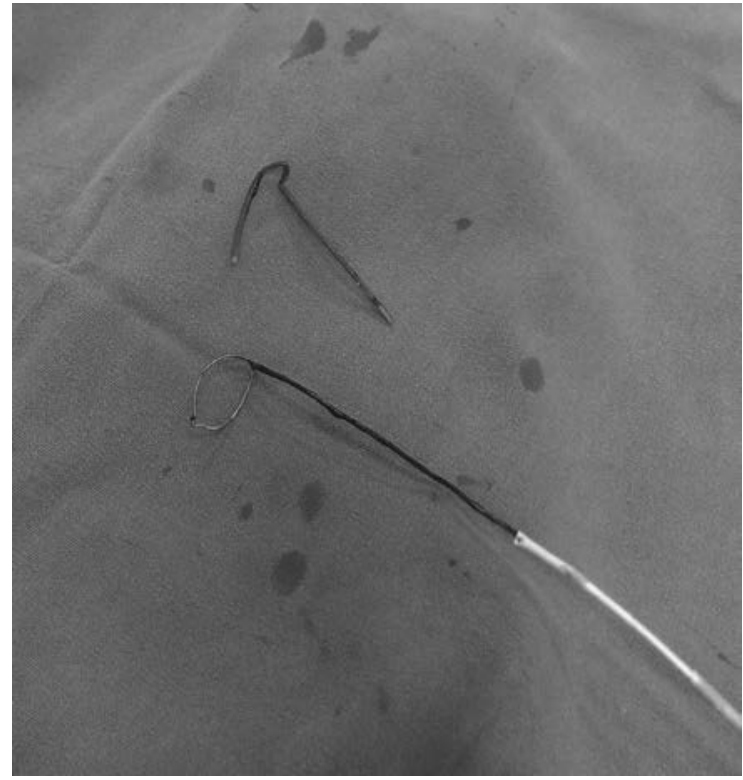

Fig. 1. Transcatheter retrieval of embolized catheter piece.

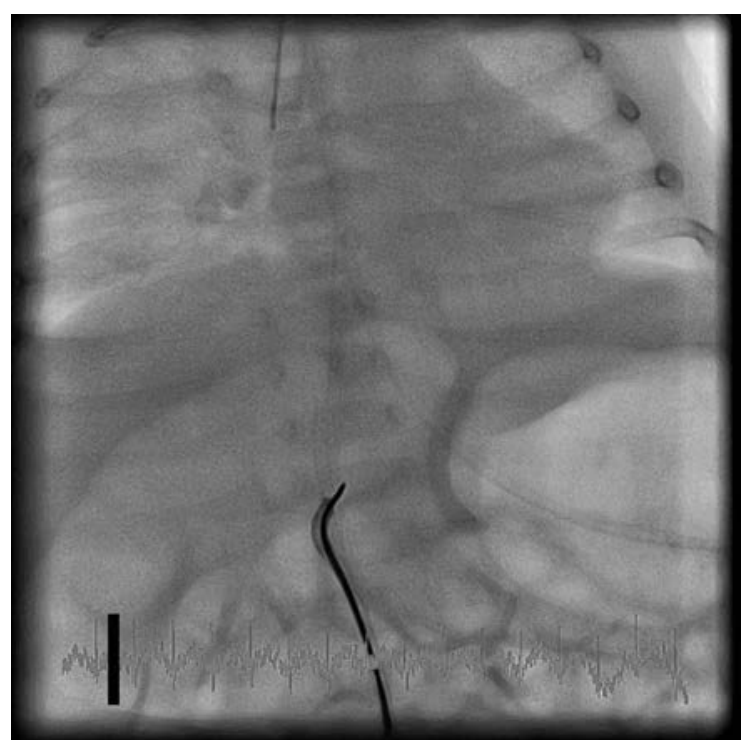

Fig. 2. Catheter piece; its lower end was caught by snare and it was removed from right femoral vein with the sheath without any complication.

unsuccessful and the patient was referred to our center. The catheter piece was stuck between hepatic vein and right upper pulmonary vein. The patient was taken to the catheter laboratory and under general anesthesia, five French sheath was percutaneously inserted in right femoral vein. A pigtail catheter was advanced to the right atrium and we tried to catch and pull the catheter piece to the right atrium but it got free of the pigtail catheter. Then another five French sheath was placed in the left femoral vein and 0.035 inch glide guide wire was placed in right atrium through the pigtail catheter. A five French guiding catheter was advanced from right femoral vein to the right atrium carrying a goose neck snare catheter. As pigtail catheter and guide wire turned around the catheter piece, guide wire was caught by snare and right-left femoral venous-loop was created, catheter piece was squeezed in between and pulled out, then it dropped to inferior vena cava: Its lower end was caught by snare and it was removed from right femoral vein with the sheath without any complication (Fig. 1 and 2). After the day of intervention, the patient was successfully extubated. The patient was discharged from the hospital on the fifth day. Later, as we examined the retrospective history of the patient, it was learned that umbilical venous catheter was inserted on the first day after birth for supportive care, and that suture was utilized for fixation and at the time sutures were cut off by scalpel and removed on the 20th day, the catheter was also cut and remained in umbilical vein. As a result of examinations made upon patient's recurrent sepsis attacks and for her prolonged respiratory distress it was learned that the catheter piece was left in umbilical vein and this was recognized 15 days later. The family of the patient was provided with information about this current work and informed consent form was signed.

\section{Discussion}

Umbilical venous catheters provide a reliable central path in order to give adequate medical care for very premature babies. Infection, bleeding, thrombosis, catheter occlusion and rupture and migration of a piece of catheter to anywhere in the body can be observed. ${ }^{1,2}$ The catheter piece may behave like foreign body and tends to form thrombosis and infection. It may also lead to many complications based on the place it has migrated such as respiratory distress, arrhythmia, cardiac perforation, pericardial effusion and liver damage. ${ }^{1,3,4}$ In this case, the catheter piece was extending from umbilical vein up to right upper pulmonary vein and the patient had findings of respiratory distress, failure to wean from mechanical ventilation and unhealed infection. 
Insertion care, and removal of umbilical venous catheters by qualified healthcare professionals reduce possible complications. During the fixation of umbilical venous catheters, different techniques are used such as banding in bridge form and suturing to skin. Scissor or scalpel are used during the removal of catheters which are sutured to skin. During the removal of sutures, movement of the patient and improper use of scissor or scalpel cause catheter to be cut or embolize into umbilical vein as was the case in our patient. Retrieval of retained umbilical artery catheter has previously been attempted using transumbilical, percutaneous and laparotomy approaches..$^{5-7}$ Although it is stated that catheter piece is removed by surgical method or umbilical venous way, there are rare cases which removal was made transcatheter by femoral venous way. ${ }^{5,6}$ Our case is unique because femoral venovenous loop was used to retrieve the stuck catheter in a small baby.

In order to minimize the complications related with umbilical venous catheters, banding in bridge format should be used during its fixation. Suturing to skin and removal by scissor and scalpel must be avoided. The place of catheters should be checked radiographically, and the catheter should be checked intermittently for complications. In this case we demonstrated that embolized catheters can be retrieved by forming venovenous loop.

\section{REFERENCES}

1. Cortwright DW. Central venous lines in neonates; a study of 2186 catheters. Arch Dis Child Fetal Neonatal Ed 2004; 89: 504-508.

2. Fusco FP, De Carolis MP, Costa S, De Rosa G, Zambraro A, Romagnoli C. Cardiac complications in preterm infants with percutaneous long lines: the importance of early diagnosis. Resuscitation 2008; 79: 506-508.

3. Jouvencel P, Tourneux P, Pérez T, et al. Central catheters and pericardial effusion: results of a multicentric retrospective study. Arch Pediatr 2005; 12: 1456-1461.

4. Sehgal A, Cook V, Dunn M. Pericardial effusion associated with an appropriately placed umbilical venous catheter. J Perinatol 2007; 27: 317-319.

5. De Carolis MP, Costa S, Polimeni V, Di Stasi C, Papacci P, Romagnoli C. Successful removal of catheter fragment from right atrium in a premature infant. Eur J Pediatr 2007; 166: 617-618.

6. Pérez-Martínez A, Bento-Bravo L, Martínez-Bermejo MA, Conde-Cortés J, Lezáun R, Egüés J. Fracture and intracardiac migration of a neonatal venous catheterextraction via the umbilical pathway. Pediatr Radiol 2002; 32: 211 .

7. Murphy KD, Le VA, Encarnacion CE, Palmaz JC. Transumbilical intravascular retrieval of an umbilical artery catheter. Pediatr Radiol 1995; 25: 178-179. 